\title{
Involvement of CsWRKY70 in salicylic acid-induced citrus fruit resistance against Penicillium digitatum
}

\author{
Bing Deng', Wenjun Wang ${ }^{1}$, Changqing Ruan ${ }^{1,2}$, Lili Deng ${ }^{1,2}$, Shixiang Yao ${ }^{1,2}$ and Kaifang Zeng ${ }^{1,2}$
}

\begin{abstract}
Penicillium digitatum causes serious losses in postharvest citrus fruit. Exogenous salicylic acid (SA) can induce fruit resistance against various pathogens, but the mechanism remains unclear. Herein, a transcriptome-based approach was used to investigate the underlying mechanism of SA-induced citrus fruit resistance against $P$. digitatum. We found that CSWRKY70 and genes related to methyl salicylate (MeSA) biosynthesis (salicylate carboxymethyltransferase, SAMT) were induced by exogenous SA. Moreover, significant MeSA accumulation was detected in the SA-treated citrus fruit. The potential involvement of CSWRKY70 in regulating CSSAMT expression in citrus fruit was studied. Subcellular localization, dual luciferase, and electrophoretic mobility shift assays and an analysis of transient expression in fruit peel revealed that the nucleus-localized transcriptional activator CSWRKY70 can activate the CsSAMT promoter by recognizing the $\mathrm{W}$-box element. Taken together, the findings from this study offer new insights into the transcriptional regulatory mechanism of exogenous SA-induced disease resistance in Citrus sinensis fruit.
\end{abstract}

\section{Introduction}

Citrus fruit is threatened by a variety of pathogens, and the decay caused by Penicillium digitatum infections accounts for most of the economic losses during postharvest storage ${ }^{1,2}$. In practice, the extensive use of artificial fungicides, including thiabendazole, sodium $o$-phenyl phenate, and imazalil, has effectively inhibited the spread of citrus postharvest disease $\mathrm{e}^{3-5}$. However, the emergence of resistant strains of pathogens and undesirable influences on the environment and human health caused by the wide use of these chemical fungicides have led to an increasing and urgent need for efficient and healthy alternatives to fungicide usage ${ }^{6}$.

Salicylic acid (SA) is one of the most important hormones in plants. Although the regulatory roles of SA in plant resistance to abiotic stress, growth, and development have been widely demonstrated, the most well known is its pivotal role in plant defense responses ${ }^{7,8}$. In flowering plants, SA is synthesized in either the cinnamic

\footnotetext{
Correspondence: Kaifang Zeng (zengkaifang@hotmail.com)

${ }^{1}$ College of Food Science, Southwest University, 400715 Chongqing, China ${ }^{2}$ Research Center of Food Storage \& Logistics, Southwest University, 400715 Chongqing, China
}

acid pathway or the chorismate pathway ${ }^{9}$. SA and its conjugates formed via methylation (methyl salicylate (MeSA)) and glycosylation (SA 2-O-b-D-glucoside) have been determined to be necessary for plant defense responses ${ }^{10}$. Apart from its positive role in host defense responses, applied SA influences the disease resistance of postharvest fruits ${ }^{11-14}$. The application of exogenous SA conferred resistance to many monocotyledonous and dicotyledonous plants to a variety of pathogenic microorganisms, such as viruses, bacteria, and fungi, and the underlying mechanisms were attributed to the deposition of callose plugs (reinforcements of plant cell walls), the synthesis of $\mathrm{H}_{2} \mathrm{O}_{2}$ (initiators of hypersensitive responses) and the accumulation of pathogenesis-related proteins (induce toxicity in microbes upon contact) ${ }^{11-14}$. For postharvest fruits, the application of exogenous SA may enhance the activity of disease resistance-related enzymes ( $\beta$-1,3-glucanase, phenylalanine, and peroxidase), induce the expression of pathogenesis-related genes, and further enhance the disease resistance of postharvest fruits ${ }^{6,12,15}$. Although many studies have been conducted to determine the mechanism of SA-induced host resistance, few studies have focused on the underlying molecular 
mechanism of the transcriptional regulation in postharvest fruit.

The WRKY gene family is one of the largest transcription factor (TF) families in higher plants ${ }^{16,17}$. WRKY TFs (WRKYs) contain a DNA-binding domain that includes one or two highly conserved WRKYGQK motifs that follow a specific zinc-finger motif in the $C$ terminus ${ }^{16,17}$. WRKYs participate in many biological processes, the best known of which is their pivotal role in plant defense responses ${ }^{18}$. According to a previous study, many WRKYs have been found to participate in SA-induced host resistance ${ }^{19-25}$. Exogenous SA can induce the expression of Arabidopsis thaliana WRKY3, WRKY72, WRKY38/62, and WRKY46 and Oryza sativa WRKY30, WRKY45, and WRKY23, among others ${ }^{19-25}$. These SA-induced WRKYs may enhance plant disease resistance by activating the transcription of defense-related genes or repressing the transcription of development-relevant genes ${ }^{26-28}$. Nevertheless, the regulatory role of WRKYs in the postharvest disease resistance of citrus fruit remains unexplored.

In this study, the effect of SA at different concentrations on citrus fruit resistance against $P$. digitatum was investigated. Transcriptome analyses on SA-treated and control citrus fruit were carried out to better understand the underlying mechanism. Based on RNA-sequencing (RNAseq) analysis, SA-responsive CsWRKY70 was identified, and the possible association of CsWRKY70 with the direct activation of CsSAMT was investigated. This work provides new information on the mechanism of exogenous SA-induced host resistance in citrus fruits.

\section{Results}

Effect of exogenous SA on the disease resistance of citrus fruit against $P$. digitatum

Decay caused by fungus accounts for the greatest loss of citrus fruit in postharvest storage ${ }^{15,29}$. In this study, we investigated the effect of different concentrations of SA on the disease incidence and lesion size of citrus fruit. As shown in Fig. 1a, the disease incidence of $2 \mathrm{mM}$ SAtreated fruits was only $34 \%$, while that of the control was $51 \%$ when measured $121 \mathrm{~h}$ post inoculation (h.p.i.). Compared with the control group, 2 and $4 \mathrm{mM}$ SA treatment inhibited the enlargement of the lesion size, and $2 \mathrm{mM}$ was the most effective SA concentration. In a previous study, the application of $2 \mathrm{mM}$ SA obviously inhibited the development of green mold, which is consistent with our study ${ }^{6}$.

Transcriptome analysis reveals the underlying mechanism of the SA-induced resistance of citrus fruit

To obtain a better understanding of the underlying mechanism of SA-induced citrus fruit resistance against $P$. digitatum, transcriptome analyses of citrus fruit 8 and 48 h.p.i. were performed. A Gene Ontology (GO) enrichment analysis showed that the differentially expressed genes (DEGs) induced by SA were enriched in many disease resistance-related gene functions (Fig. 2a). In addition, we analyzed the differential expression levels of genes correlated with immune responses (detailed information for the DEGs involved in immune responses is listed in Supplementary Table S2). As shown in Fig. 2b, several genes correlated with PAMP (pathogen-associated molecular pattern)-triggered immunity (PTI) were responsive to SA 8 h.p.i., including genes encoding the respiratory burst oxidase-like protein (which plays a pivotal role in the accumulation of reactive oxygen species) and cyclic nucleotide-gated ion channel 1 protein, which mediate the signal transduction of plant PTI. In addition to PTI-related DEGs, several genes encoding R proteins, which are critical for recognizing fungal effector proteins, were differentially expressed in the later stage (48 h.p.i.). Remarkably, two genes encoding salicylate carboxymethyltransferase (SAMT) were found to be differentially expressed in SA-treated citrus fruit (the $\log _{2}$ fold change of these genes is listed in Supplementary Table S2). In higher plants, the methyl esterification of SA can be catalyzed by SAMT to produce MeSA ${ }^{30,31}$.

MeSA acts as a phloem-mobile signal to establish systemic acquired resistance ${ }^{32,33}$. To investigate whether the accumulation of MeSA is influenced by exogenous SA, the changes in MeSA content in the citrus fruit during the storage period were measured. As shown in Fig. 3c, the endogenous MeSA content was notably $(P<0.05)$ enhanced by the SA treatment. In particular, at 8,24 , and 72 h.p.i., the MeSA content in the SA-treated fruits was over 2-, 4-, and 3.5-fold higher than that in the controls at 8,24 , and $72 \mathrm{~h}$, respectively. The qRT-PCR analysis showed that the expression levels of CsSAMTs were largely consistent with the changes in MeSA. These results suggest that exogenous SA can induce the synthesis of MeSA in citrus fruit.

\section{Characterization of CsWRKY70}

WRKYs perform critical roles in host disease resistance to biotic stresses, and many WRKYs have been identified as important regulators of SA-induced host resistance ${ }^{18}$. Given that exogenous SA induces MeSA accumulation, we were interested in the underlying transcriptional mechanism. We conducted transcriptome analysis of citrus fruit in response to different stimulants (SA and other phytohormones), and the results showed that CsWRKY70 was specifically responsive to exogenous SA (unpublished data). As shown in Fig. 4a, CsWRKY70 was significantly upregulated at both time points (the $\log _{2}$ fold change at 8 and 48 h.p.i. was 2.3 and 3.4, respectively, as shown in Supplementary Table S2). To confirm the specificity of CsWRKY70 in SA-induced citrus fruit resistance against $P$. digitatum, the expression of CsWRKY70 was measured by reverse transcription- 

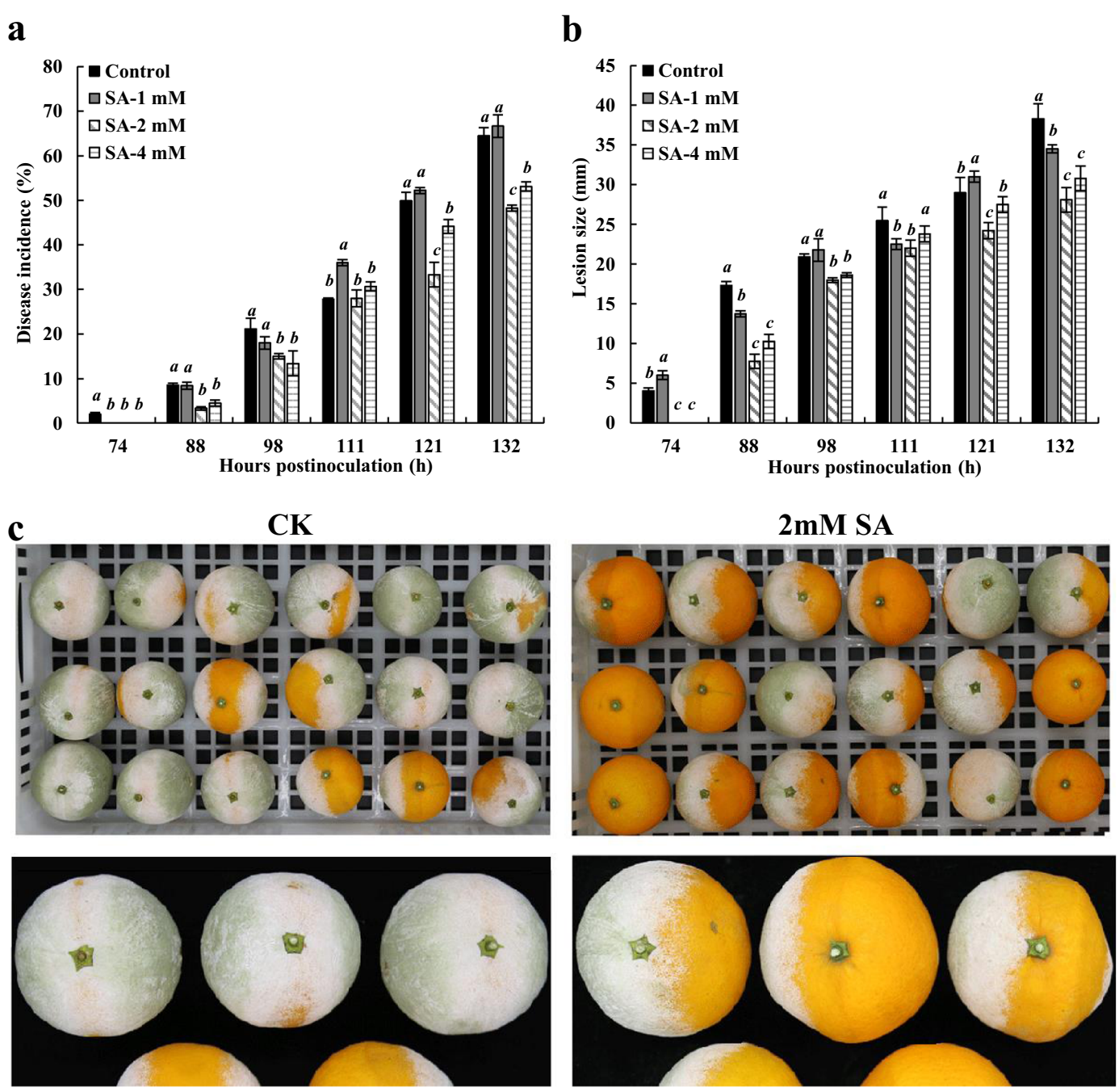

Fig. 1 Effects of exgenous SA on the disease incidence and lesion size of citrus fruit caused by $P$. digitatum. a Changes of disease incidence. b Changes of lesion size. These two indicators were measured $74,88,98,111,121$, and 132h post inoculation. Data represent the mean \pm s.e.m. of three biological replicates $(n=3)$. c Appearance of the control and $2 \mathrm{mM}$ SA-treated citrus fruit at the late stage of disease

quntitative PCR (RT-qPCR (Fig. 4b). In accordance with its trend in the transcriptome data, the CsWRKY70 expression level upon SA treatment increased after pathogen infection, which implies that CsWRKY70 participated actively in the SA-induced immune responses of the citrus fruit.

According to the RNA-seq and C. sinensis genome databases (http://citrus.hzau.edu.cn/ orange), the fulllength CsWRKY70 was cloned. Through a homology search, we found that its sequence shared the highest identity with Solanum lycopersicum SIWRKY70 (4-c). CsWRKY70 contains a complete open-reading frame (ORF) of $984 \mathrm{bp}$, which encodes a protein of 328 amino acids, and the predicted molecular weight and isoelectric point of CsWRKY70 were 36.7 and $6.32 \mathrm{kDa}$, respectively. The amino acid sequence analysis revealed that CsWRKY70 presented one highly conserved zinc-finger containing the WRKY DNA-binding domain (60 amino acids, extending from 141 to 200) in the C-terminal region, and the zinc-finger pattern was $\mathrm{C}-\mathrm{X} 7-\mathrm{C}-\mathrm{X} 23-\mathrm{H}$ $\mathrm{X} 1-\mathrm{C}$ (Fig. 4d). According to the common classification methods based on A. thaliana, WRKYs in plants can be classified into three groups (I, II, and III) based on their number of WRKY domains and the zinc-finger-like motif features ${ }^{18}$. The phylogenetic tree constructed with CsWRKY70 and model plant WRKYs showed that CsWRKY70, SIWRKY70, and AtWRKY70 had the same pattern of conserved group III WRKY proteins.

\section{Analysis of the subcellular localization and transcriptional activity of CsWRKY70}

TFs are produced in the cytoplasm, and the transport of TFs from the cytoplasm to the nucleus mediated by the 
a

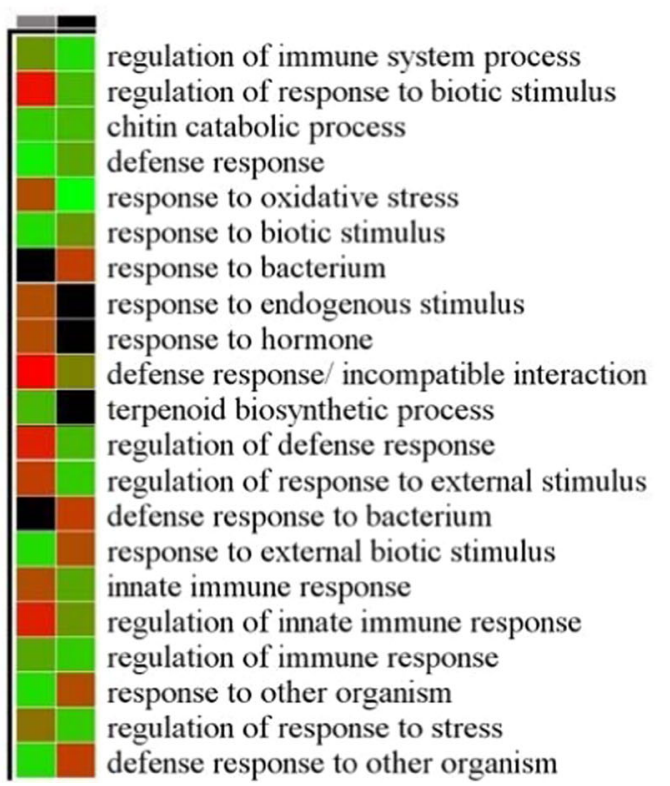

b

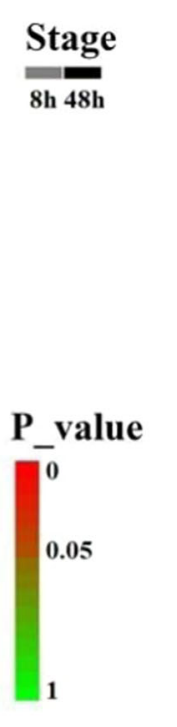

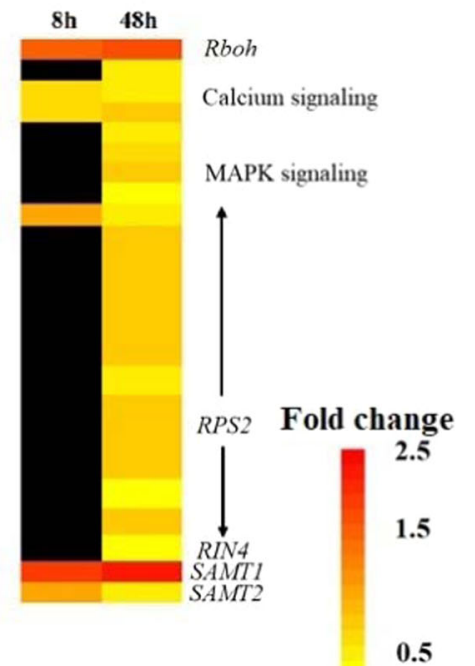

0.5

Fig. 2 Analysis of DEGs related to disease resistance. a Heat map of Gene Ontology terms related to disease resistance as identified for upregulated genes. The red cubes indicate high enrichment factors for the corresponding gene functions, and the black cubes indicate that the corresponding gene functions are not enriched. $\mathbf{b}$ Heat map of the genes related to disease responses. Red represents a high fold change, and black represents genes that are not differentially expressed

recognition nuclear localization signals (NLSs) by transporter proteins is a necessary step for posttranslational regulation $^{34,35}$. A predicted NLS at the $\mathrm{N}$ terminus (119-132) implies that CsWRKY70 is translocated into the nucleus to regulate gene transcription (Fig. 4d). To investigate the subcellular location of CsWRKY70, the coding sequence of CsWRKY70 was fused with the gene encoding green fluorescent protein (GFP) in a pEAQ plasmid, and the construct was transiently expressed in tobacco leaves. As shown in Fig. 5, the GFP fluorescence of the CsWRKY70-GFP fusion protein was present only in the nucleus, while in the positive control, the GFP signal was observed throughout the cytosol and nucleus. The results indicated that CsWRKY70 was localized to the nucleus.

Depending on the transcriptional regulation ability, TFs can be categorized as transcriptional activators or transcriptional repressors ${ }^{36}$. A dual-luciferase reporter system was used to examine the transcriptional activity of CsWRKY70 in tobacco leaves. Firefly luciferase (LUC) fused with five GAL4 DNA-binding elements plus a TATA box and Renilla luciferase (REN) driven by the $35 \mathrm{~S}$ promoter constituted the reporter, and CsWRKY7O fused with the GAL4 DNA-binding domain in the $\mathrm{pBD}$ vector was used as the effector (Fig. 5b). As shown in Fig. 5c, the LUC/REN ratios of pBD-VP16 (transcriptional activator control) and pBD-CsWRKY70 were all significantly higher than those of $\mathrm{pBD}$. These results indicated that
CsWRKY70 was a transcriptional activator localized in the nucleus.

\section{CsWRKY70 specifically binds to the CSSAMT promoter and activates the expression of CSSAMT genes}

The WRKY domain can bind to target genes by recruiting the W-box in the promoter region (cis-acting element $)^{18}$. In a previous study, many W-box sequences were specifically bound to WRKY TFs, and the combination was essential for the expression of target genes ${ }^{37-39}$. A motif scan of the CSSAMT genes revealed W-boxes in the promoters of both CSSAMT genes, which implied that the expression of CSSAMT might be regulated by WRKY TFs. Remarkably, qRT-PCR revealed that the CsWRKY70 and CSSAMT genes had similar expression patterns during the storage period. Based on the above-mentioned results, we inferred that CSSAMTs might be the target genes of CsWRKY70. To confirm this hypothesis, an electrophoretic mobility shift assay (EMSA) was conducted to verify the binding specificity of the recombinant CsWRKY70 to the CsSAMT promoter. The recombinant His-CsWRKY70 protein was efficiently expressed in the Escherichia coli expression system and purified with $\mathrm{Ni}$ NTA chromatography (Fig. 6a). As shown in Fig. 6b, c, the band shifts were observed when the CsWRKY70 protein was incubated with each biotin-labeled probe (containing W-boxes), which indicated that CsWRKY70 can directly bind to these sequences. The CsWRKY70 can bind to the 

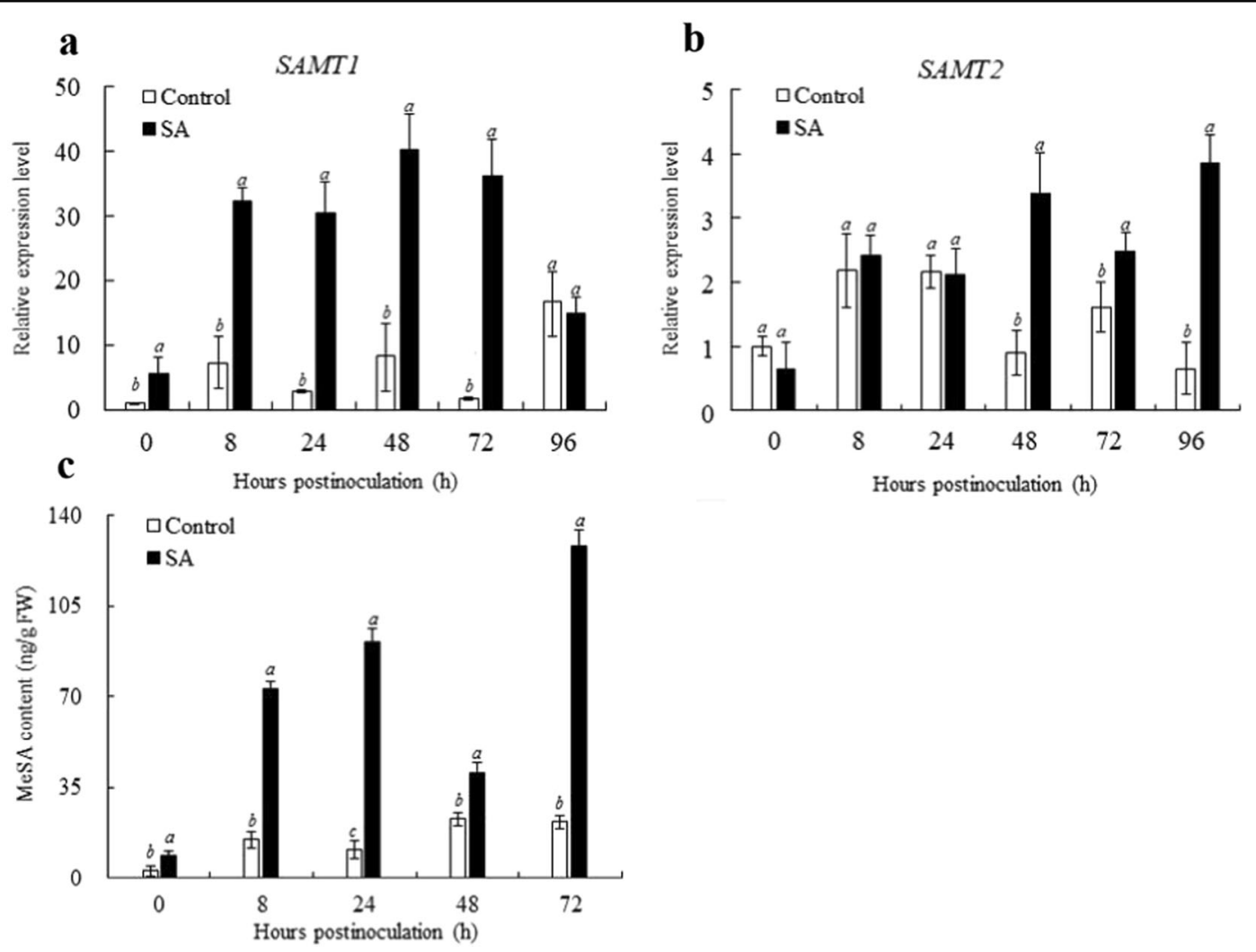

Fig. 3 Effects of exogenous SA on the synthesis of MeSA upon pathogen inoculation. a The expression level of CSSAMT1. b The expression level of CSSAMT2. c Changes in the endogenous MeSA content. Citrus fruits were treated with $2 \mathrm{mM} \mathrm{SA}$ or water (control) following harvest, and then $10 \mu \mathrm{L}$ of $P$. digitatum spores $\left(5 \times 10^{4}\right.$ spores $/ \mathrm{mL}$ in suspension) was inoculated, and the fruits (incubated at $25^{\circ} \mathrm{C}$ in $90-95 \%$ relative humidity) were sampled 0, 8, 24, 48, 72, and 96 h.p.i. Differential expression levels were determined by qRT-PCR. Citrus sinensis actin (XM_006464503) was employed as the housekeeping gene, and the expression level of each CSSAMT gene was calculated as the ratio relative to the control sample at 0 h.p.i. (set as 1). Each value represents the mean \pm s.e.m. of three replicates $(n=3)$

biotin-labeled probes and the corresponding unlabeled probe, with which it competes, but not to the corresponding unlabeled mutant probe (containing the mutated W-box). These results suggested that CsWRKY70 specifically was bound to the CsSAMT promoter.

CsWRKY70 is a transcriptional activator, and it can specifically bind to the CSSAMT promoter. These results imply that CsWRKY70 may positively regulate the expression of CsSAMTs. To verify this supposition, the ability of CsWRKY70 to activate the transcription of the CSSAMT genes was detected through transient dual-luciferase assays. As shown in Fig. 6d, the CsSAMT1 and CSSAMT2 promoters were inserted into a pGreenII 0800-LUC vector to serve as reporters. CsWRKY70 was inserted into a $\mathrm{PEAQ}$ vector to serve as an effector, and pEAQ was used as a control. As shown in Fig. 6e, the transient expression of CsWRKY70 significantly enhanced the LUC/REN ratio of the reporters containing CsSAMTs relative to that of the corresponding empty control.
Due to the long juvenile period of citrus, it is very difficult to verify the function of CsWRKY70 in vivo by using gene-silencing technology or stable overexpression lines. To better illustrate the regulatory effects of CsWRKY70 on the CSSAMT genes, transient overexpression of CsWRKY70 in citrus fruit peels was conducted. As shown in Fig. 6f, the transient overexpression of CsWRKY70 resulted in the expression of CSSAMT1 and CsSAMT2 3 days post infiltration. These results suggested that CsWRKY70 can activate the expression of CsSAMTs.

\section{Discussion}

Penicillium digitatum is one of the most destructive postharvest pathogens of citrus fruit ${ }^{2}$. Some chemical treatments, such as 2,4-D, thiabendazole and imazalil, have been widely used to inhibit the spread of the disease $^{5}$. However, considering the potential hazards to human health and the environment of the abovementioned methods, it is necessary to find an environmentally friendly and effective method ${ }^{6}$. The induction 
$\mathbf{a}$

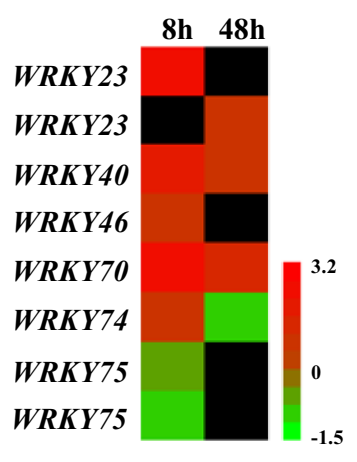

c

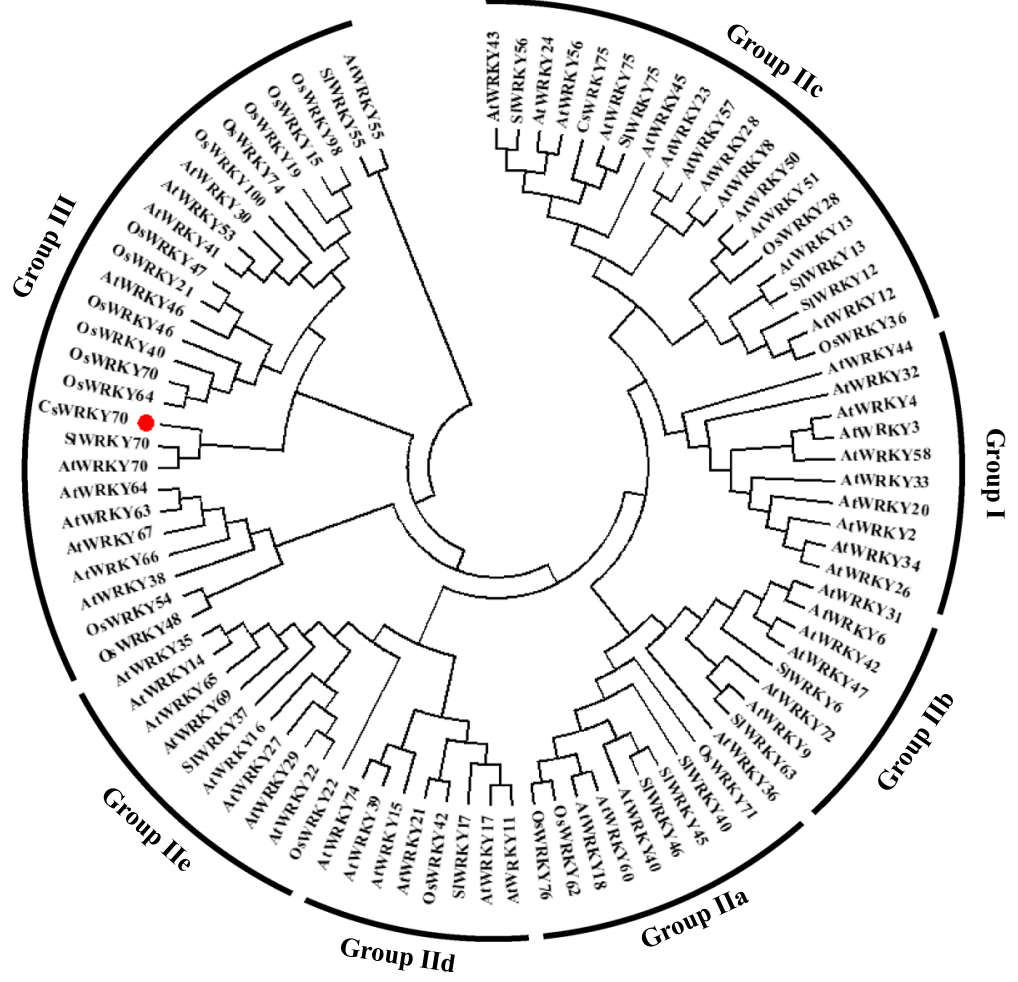

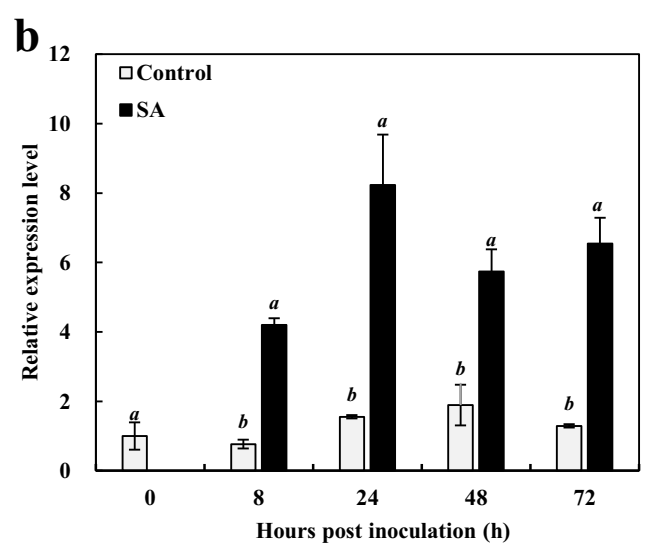

d

CSWRKY70 : ESSKSSTVKIRRGCYKRRKCAESW|'EHSSTITD-DGHAWRKYGQIVIINARFPFNYERCTHKFDCGC : 174 SIWRKY81 : GSCKSS---DRRGCYKRRKTSESDIKESSDIVD-DGHAWRKYGQKOIINSTYPRHYERCTHKYDOKC : 143 S1WRKY70 : LKKKKKEKQDRRGCYKRRKTSGSWMRESATNN--DGCAWRKYGQKKIINSKYPROYYRCTHKYDOEC : 149 AtWRKY70 : RK-RLGEVKGKRGCYKRKKRSETCTIESTIIE--DAF SWRKYGQKEIINAKFPRSYERCTHKYTCGC : 153 AtWRKY54 : KKKRLGVGKGKRGCYTRKTRSHARIVEAKSSE--DRYAWRKYGQKEIINTTFPRSYLRCTHKPTCGC : 185 SIWRKY55 : SSORQRTRCRTGDRDRRTLRVGAPRMGNLETPPEDGYTWRKYGQKEIIGSREPFAYYRCTHQKLYHC : 180

$$
\mathrm{R} \quad \mathrm{D} \underset{\text { WRKYGQK IL PRY RCTH }}{+}+
$$

CSWRKY70 : CASKQVQRIQEEETP-IHRTTYYGRHTCKSLIKSSQLMLDSTTSDQCPMISFGSAHITE--------- : 231 S1WRKY81 : ¿ASKQVQKIQDNPQ-RFRTTYYGHHTCKAFPRVSQIILDSQIDGNSNYISFDQNHT---------- : 198 SIWRKY70 : RA HKCVQIIQENEIIMYHTTYF ENHTCN------PTKIPKHTYNNHAN VKHSDSTV----------- : 199 AtWRKY70 : KATKCVQKVELEEK-MESITYI ENHTCNTN----AETPKSKTCDHHDEIFMDSE------------ : 202 AtWRKY54 : KATKQVQKQDQDSE-MEQITIGYHTCTAN----DQT-HAKTEPFDQEIIMDSEKTLAASTAQNHVN : 246 SIWRKY55 : PAKKQVQRTDNDFY-VFEVTYRSOHTOYMSATAPTVPPPSAEEMTHKTTTTLLPPAPLLLPPP----- : 241 $\mathrm{T}$ KCVQ
++

Fig. 4 (See legend on next page.) 
(see figure on previous page)

Fig. 4 Characterization of CsWRKY70 in citrus fruit disease resistance against $P$. digitatum following exogenous SA treatment. a Differentially expressed transcription factor genes. b Transcript level of CSWRKY70 in citrus fruit with disease resistance against $P$. digitatum after exogenous SA treatment. c Phylogenetic tree of CsWRKY70, O. sativa, S. lycopersicum, and A. thaliana WRKYs. CsWRKY70 (red circle), along with A. thaliana AtWRKY70, S. lycopersicum SIWRKY70, O. sativa OsWRKY70, and O. sativa OsWRKY64 clusters in group III. d Relationships between CsWRKY70 and other plant WRKY family proteins. Identical amino acid sequences in different WRKYs are represented by black shading. The WRKY motif is underlined, the nuclear localization signal is marked in a red box, and the zinc-finger structure is indicated by asterisks

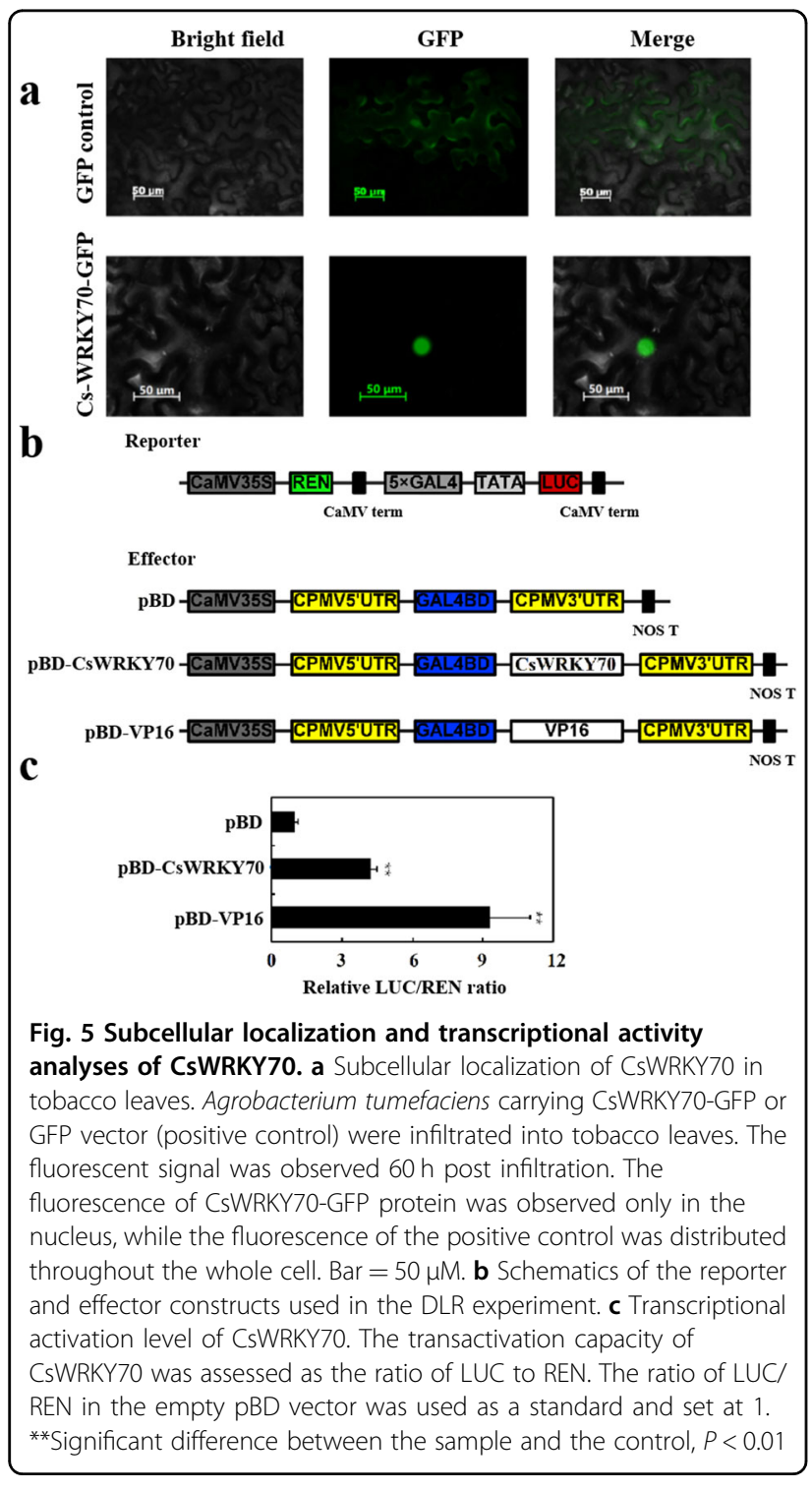

of host resistance is a promising alternative to avoid the negative side effects of chemical treatments ${ }^{6}$. As a pivotal signaling molecule, SA plays pivotal roles in plant immune responses against a variety of biotic stresses ${ }^{8}$. Moreover, it has been validated that exogenous SA has positive effects on inducing the host resistance of many plants and postharvest fruits ${ }^{6,11,15,40}$. However, the applicability of SA for inducing citrus fruit disease resistance and the underlying molecular mechanism are still unclear.

Exogenous SA can induce natural resistance of citrus fruit A previous study confirmed that the application of $2 \mathrm{mM}$ SA significantly decreased the decay of Satsuma mandarin (Citrus unshiu) fruit during postharvest storage $^{6}$. In the present study, the effect of different concentrations of SA on the disease resistance of $C$. sinensis fruit against $P$. digitatum was examined. The results suggested that a $2 \mathrm{mM} \mathrm{SA}$ treatment is a potent method to inhibit the occurrence of green mold. Transcriptome analyses showed that SA treatment can enhance host immune responses against $P$. digitatum by inducing the expression of various disease resistance genes. Remarkably, two CSSAMT genes, which are essential for the formation of MeSA, were identified as differentially expressed in SA-treated citrus fruit. More importantly, the endogenous MeSA level in the SA-treated samples was significantly higher than that of the control fruit after pathogen infection. These results implied that exogenous SA can induce the accumulation of MeSA in citrus fruit. In a previous study, $0.2 \mathrm{mM}$ SA was applied to transformed Atropa belladonna roots induced the accumulation of MeSA and methyl-o-methoxy-benzoate ${ }^{41}$. Further research revealed that AbSAMT1 mRNA began to be expressed $12 \mathrm{~h}$ post exposure, and steady expression continued over $144 \mathrm{~h}^{41,42}$. The accumulation of MeSA might contribute to SA-induced disease resistance.

\section{CsWRKY70 participates in the transcriptional regulation of SA-induced disease resistance}

WRKY TFs are best known for their transcriptional regulation of plant disease resistance ${ }^{18}$. In previous studies, the involvement of five WRKY III subfamily AtWRKYs in A. thaliana immune responses was verified, and all of these TFs (AtWRKY38, AtWRKY46, AtWRKY53, AtWRKY62, and AtWRKY70) were induced by either Pseudomonas syringae or exogenous $\mathrm{SA}^{20,43}$. Further research showed that AtWRKY70 plays a synergistic role in the SA-signaling-mediated immune responses of $A$. thaliana, which suggests the possible involvement of CsWRKY70 in the SA-induced disease resistance of citrus fruit ${ }^{43}$. 


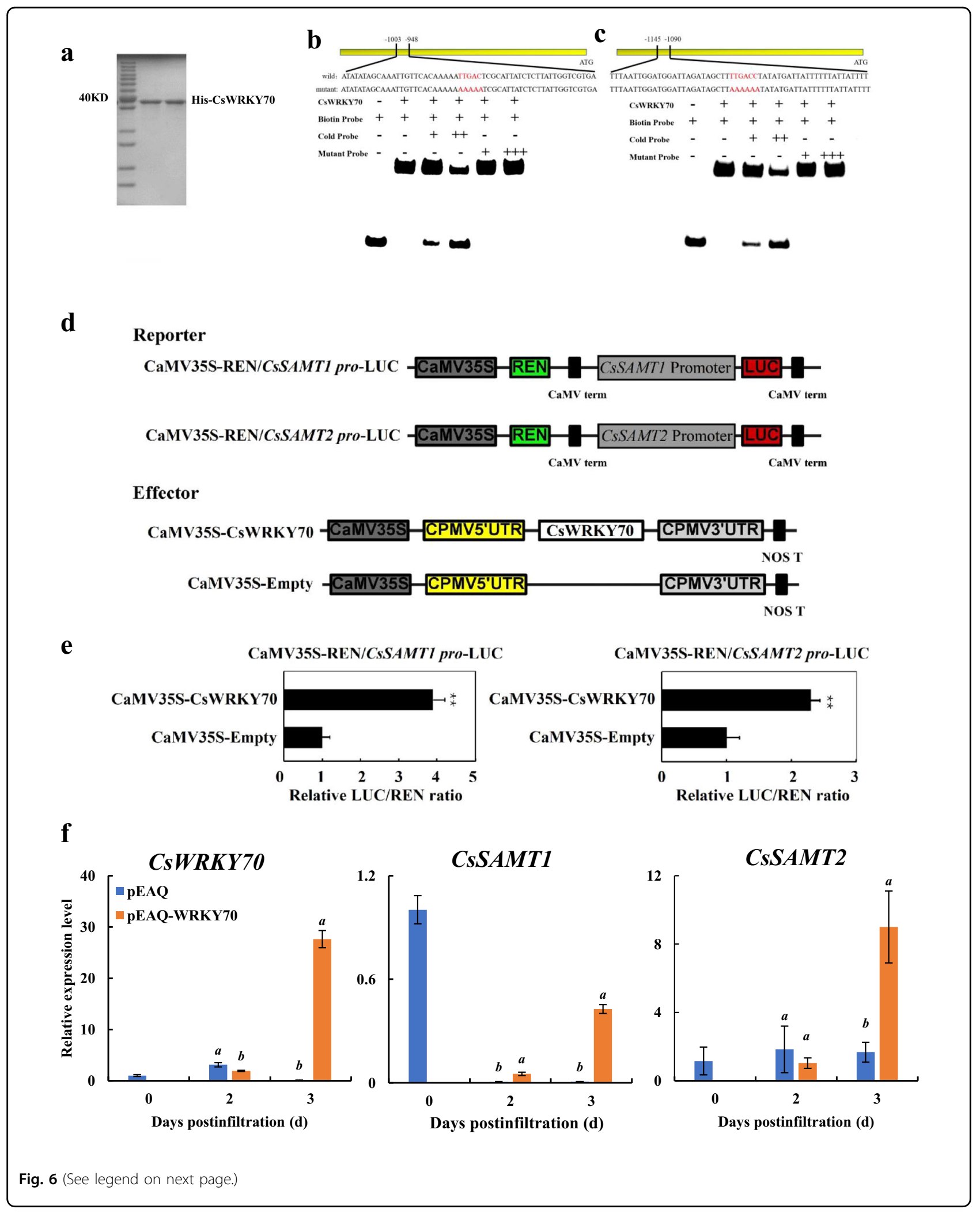


(see figure on previous page)

Fig. 6 CsWRKY70 specifically binds to the CSSAMT promoter and activates the expression of CsSAMTs. a SDS-PAGE was conducted to detect the purification of the recombinant CsWRKY70 protein. b, c EMSA. Biotin-labeled probe or mutant probe was incubated with His-CsWRKY70 protein, and the mixtures were separated on a $6 \%$ native polyacrylamide gel. The symbols + and ++ indicate increasing amounts of unlabeled probe used for competition with or increasing the amounts of mutant probe used to test the binding specificity. $\mathbf{d}$ Schematics of the reporter and effector constructs used in the DLR experiment. The CSSAMT promoter was inserted into a PGREENII 0800-LUC vector (Kpnl and Ncol) as a reporter, and the full-length CSWRKY7O was fused to a PEAQ vector (Agel and Xhol) as an effector. Each pair of effector and reporter plasmids was transformed into an $A$. tumefaciens strain and then transiently expressed in tobacco leaves (Nicotiana benthamiana). e CSWRKY70 transactivates the CSSAMT promoters. Activation was indicated by the ratio of LUC to REN. The ratio of LUC/REN of the empty vector plus promoter was used for calibration and set to 1. Each value represents the mean \pm s.e.m. $(n=5)$. ${ }^{*}$ Statistically significant differences at $P<0.01$. $\mathbf{f}$ Effect of transient CsWRKY70 expression on endogenous MeSA biosynthesis genes (CSSAMTS). Error bars indicate standard errors (SEs) from three replicates

In a previous study, the transcription of $A t W R K Y 70$ was activated by exogenous $\mathrm{SA}$ and inhibited by exogenous $\mathrm{JA}^{44}$. Further studies confirmed that the constitutive overexpression of AtWRKY70 induced the constitutive expression of SA-induced pathogenesis-related genes (PR1) and, in contrast, suppressed AtWRKY70-activated JA-responsive genes (PDF1.2 $)^{44,45}$. These results suggest that AtWRKY70 acts as an activator of SA-induced genes and a repressor of JA-responsive genes ${ }^{44,45}$. In addition, the expression of PtrWRKY89 (the homolog of AtWRKY70) was induced by exogenous SA in Populus trichocarpa $^{46}$. Moreover, the overexpression of PtrWRKY89 induced the expression of the SA-signaling pathway-responsive $P R s^{46}$. Notably, the overexpression of AtWRKY70 or its homologous genes did not cause changes to the SA levels or to SA biosynthesis genes ${ }^{45-47}$. These results suggested that WRKY70 is actively involved in SA-mediated disease resistance and acts downstream of the SA-dependent signaling pathway.

In this study, we found that CsWRKY70 is a group III WRKY TF. Further research showed that the nucleuslocalized transcriptional activator CsWRKY70 can specifically bind to two CSSAMT promoters to activate the expression of the CSSAMT genes. This result provides a new basis to elucidate the underlying mechanism of exogenous SA-induced disease resistance in citrus fruit.

\section{Materials and methods}

\section{Plant material and postharvest treatment}

Mature citrus fruit $(C$. sinensis L. Osbeck cv. Jincheng $447 \#$ ) was harvested from an orchard in the Beibei district of Chongqing, China. Fruits of uniform size were selected and randomly divided into two groups for further experiments. For SA treatments, citrus fruits were submerged in 1,2 , or $4 \mathrm{mmol} / \mathrm{L} \mathrm{SA}$ aqueous solutions containing $0.1 \%$ Tween-80 for $30 \mathrm{~min}$. Citrus fruits dipped in distilled water containing $0.1 \%$ Tween- 80 for $30 \mathrm{~min}$ constituted the control. Each treatment was performed with three biological replicates with $\sim 50$ fruits in each replicate. Both the control and SA-treated citrus fruits were then rinsed with distilled water and subsequently air-dried at room temperature. After $24 \mathrm{~h}$, all the fruits were wounded at two opposite points near the equator (3$\mathrm{mm}$ wide $\times 3$ - $\mathrm{mm}$ deep), and then $10 \mu \mathrm{L}$ of a $P$. digitatum suspension $\left(5 \times 10^{4}\right.$ spores $\left./ \mathrm{mL}\right)$ was inoculated at each point. All fruits were then packed in plastic bags separately and stored at $25^{\circ} \mathrm{C}$ and $90-95 \%$ relative humidity. The disease incidence and lesion size were recorded every $12 \mathrm{~h}$ according to a previous study ${ }^{48}$. Immediately after spore suspension was inoculated and after 8, 24, 48, 72, and $96 \mathrm{~h}$ of storage, the pericarp $(8 \mathrm{~mm}$ around the inoculation site) of three replicates with five fruits were collected for further analysis.

\section{RNA isolation and RNA-seq}

Approximately $1 \mathrm{~g}$ of sample from each sampling point was used to extract total RNA following the protocol described by Bugos et al. ${ }^{49}$. The extraction buffer was modified from the original and consisted of $100 \mathrm{mmol} / \mathrm{L}$ Tris, $0.2 \mathrm{mmol} / \mathrm{L} \mathrm{NaCl}, 15 \mathrm{mmol} / \mathrm{L}$ EDTA, $0.5 \%$ sodium dodecyl sulfate (SDS), and 1\% $\beta$-mercaptoethanol.

Control samples were selected for RNA-seq 0, 8 and 48 h.p.i., and SA-treated samples were selected for RNAseq 8 and 48 h.p.i. The RNA-seq and bioinformatics analyses were conducted by Novogene (Beijing, China). The method use for the library construction, RNAsequencing and RNA-seq data analysis were consistent with comparable methods described in a previous study ${ }^{48}$.

\section{CDNA synthesis, quantitative real-time PCR (RT-qPCR) and gene cloning}

Approximately $1.0 \mu \mathrm{g}$ of RNA was used to produce cDNA with an iScript gDNA Clear cDNA synthesis kit (1725034, Bio-Rad, USA). RT-qPCR was performed on a CFX96 real-time PCR system (Bio-Rad, USA) with iTaq Universal SYBR Green Supermix (1725121, Bio-Rad, USA). The PCRs and thermal cycling conditions were consistent with methods described in a previous study ${ }^{48}$. Citrus sinensis actin (XM_006464503) was used as a reference gene, and the relative expression quantity of each gene was calculated by the $2^{-\Delta \Delta C t}$ method $^{50}$.

Based on the C. sinensis genome database (http://citrus. hzau.edu.cn/orange/), the coding sequence containing the intact ORF of Cs WRKY7O was verified by PCR and cloned 
into a T cloning vector. ClustalW and GeneDoc software was used for multiple alignment assays. A phylogenetic tree consisting of WRKY proteins was constructed using the MEGA6.0 software.

\section{Genomic DNA extraction and promoter cloning}

Genomic DNA was extracted from young citrus leaves using a plant genomic DNA kit (DP305, TIANGEN). With genomic DNA as a template, the promoter sequences containing cis-elements of CsSAMTs were amplified by PCR. The online JASPAR database (http:// jaspar.genereg.net/) was used to analyze the cis-element motifs in the promoters.

\section{Quantification of endogenous MeSA content}

The extraction and quantification of endogenous MeSA content were based on the method described in a previous study $^{51}$. For endogenous hormone extraction, $\sim 1.0 \mathrm{~g}$ of citrus peels (three replicates) were ground into powder in liquid nitrogen and homogenized in $1 \mathrm{~mL}$ of extraction buffer (2-propanol: $\mathrm{H}_{2} \mathrm{O}$ :concentrated $\mathrm{HCl}=2: 1: 0.002$ ) for $1 \mathrm{~h}$ at $4{ }^{\circ} \mathrm{C}^{51}$. Endogenous MeSA was quantified by highperformance liquid chromatography-mass spectrometry using an Agilent 6460 Triple Quad system with an Agilent-XDB C18 column $(2.1 \mathrm{~mm} \times 150 \mathrm{~mm})$. Samples were injected onto the reversed-phase column using a binary solvent system composed of distilled water with $0.1 \%$ (vol/ vol) formic acid (A) and with methanol with $0.1 \%$ (vol $/ \mathrm{vol})$ formic acid (B) as a mobile phase. The oven (holding high-performance liquid chromatography column) temperature and solvent flow rate were $40^{\circ} \mathrm{C}$ and $0.3 \mathrm{~mL} / \mathrm{min}$, respectively. The mass spectrometer was operated in the positive mode using the following source settings: capillary voltage, $130 \mathrm{~V}$ (electrospray ionization (ESI)); desolvation temperature, $600^{\circ} \mathrm{C}$; and source temperature, $150^{\circ} \mathrm{C}$. Argon was used as the collision gas at a flow rate of $0.15 \mathrm{~mL} / \mathrm{min}$. MRM mode was used to monitor the precursor-to-product ion transition with a $50 \mathrm{MS}$ dwell time, and the precursor ions were fragmented with a collision energy of $23 \mathrm{~V}$, and products in the range $120-153 \mathrm{~m} / z$ were scanned. The ultra-performance liquid chromatography-ESI-tandem mass spectrometry system control, data acquisition, and data analyses were performed with Agilent MassHunter Workstation software. Seven concentration points, 100, 200, 300, 500, 600, 800, and $1000 \mathrm{ng} / \mathrm{mL}$, were used to establish calibration curves (shown in Supplementary Fig. S1).

\section{Subcellular localization}

Using the recombinant $\mathrm{T}$ plasmid as a template, the intact coding sequence without the stop codon for CsWRKY70 was amplified by using specific primers and then fused to a pEAQ-GFP vector (AgeI). The recombinant plasmid (pEAQ-CsWRKY70-GFP) was introduced into an A. tumefaciens strain (EHA105) and then transiently expressed in 5-week-old tobacco ( $N$. benthamiana) leaves according to the method of a previous study ${ }^{36}$. In addition, A. tumefaciens harboring a pEAQ-GFP plasmid was transiently expressed in tobacco leaves as the control. The GFP signal was visualized with an Axioskop 2 Plus fluorescence microscope (Zeiss, Jena, Germany) 48-60 h post infiltration.

\section{Transient transcriptional activation assay}

By using a dual-luciferase transient expression system, the transcriptional activity of CsWRKY70 and the regulatory role of CsWRKY70 on target promoters were investigated. For the assay of CsWRKY70 transcription activity, the full-length sequence without the stop codon for CsWRKY70 was recombined to a pBD vector (StuI) as an effector, and the modified PGREENII 0800-LUC vector was adopted as the reporter, which contained firefly luciferase and was driven by the CaMV35S promoter with a 5-GAL-binding element and Renilla luciferase driven by CaMV35S. To study the regulatory role of CsWRKY70 on the CsSAMT promoters, full-length CsWRKY70 was fused to a pEAQ vector (AgeI and XhoI) as an effector, and the CSSAMT1 and CSSAMT2 promoters were fused separately to the PGREENII 0800-LUC vector (KpnI and NcoI) as the reporter.

All the effector plasmids were transferred into $A$. tumefaciens (EHA105), and all the reporter plasmids were transferred into A. tumefaciens (EHA105 (pSOUP)). The cultures were adjusted to an OD600 of 0.6 with an infiltration buffer (150 $\mathrm{mM}$ acetosyringone; $10 \mathrm{mM} \mathrm{MES}$; and $10 \mathrm{mM} \mathrm{MgCl}, \mathrm{pH} 5.6$ ). After a 3-h incubation at $25^{\circ} \mathrm{C}$, the effecter and corresponding reporter in A. tumefaciens were mixed at a proportion of 9:1, and the mixtures infiltrated into tobacco ( $N$. benthamiana) leaves. The plants were cultivated in a glasshouse for $60-72 \mathrm{~h}$, and the LUC and REN activities were assayed with a dualluciferase assay kit (Promega).

\section{Electrophoretic mobility shift assay}

The full-length CsWRKY7O was fused to a pCold I vector $(K p n \mathrm{I}$ and $P s t \mathrm{I})$ to construct the His-CsWRKY70 expression vector, and the recombinant plasmid was transformed into E. coli strain BM Rosetta (DE3). HisCsWRKY70 was induced by $0.1 \mathrm{mM}$ isopropyl $\beta$-D-1thiogalactopyranoside at $15^{\circ} \mathrm{C}$ for $24 \mathrm{~h}$, and the recombinant protein was purified by the elution of gradient imidazole-containing buffers. SDS-polyacrylamide gel electrophoresis was used to evaluate the efficiency of the purification process. Probes containing a W-box in the promoters of CSSAMT were end labeled with a biotin 3'end DNA labeling kit (Thermo Scientific, 89818). EMSA was performed using a LightShift chemiluminescent EMSA kit (Thermo Scientific, 20148). 


\section{Analysis of the transient expression of the CSSAMT genes in citrus fruit peel}

By using the pEAQ-CsWRKY70 construct, a transient expression experiment was conducted in citrus fruit peel (C. sinensis). The culture of $A$. tumefaciens (EHA105) carrying pEAQ-CsWRKY70 was adjusted to an OD600 of 0.5 with an infiltration buffer $(150 \mathrm{mM}$ acetosyringone; $10 \mathrm{mM} \mathrm{MES}$; and $10 \mathrm{mM} \mathrm{MgCl}_{2}, \mathrm{pH}$ 5.6). After $3 \mathrm{~h}$ of incubation at $25^{\circ} \mathrm{C}$, the culture was infiltrated into citrus peels. The citrus fruits were stored at $25^{\circ} \mathrm{C}$ for $72 \mathrm{~h}$, and the peels were collected every $24 \mathrm{~h}$ to detect the expression level of CsWRKY70 and CsSAMT genes.

\section{Acknowledgements}

This work was supported by the National Natural Science Foundation of China (Grant numbers 31772027 and 31972126).

\section{Author contributions}

K.Z. conceived and supervised the project; B.D. and K.Z. designed the experiments; B.D. performed most of the experiments, analyzed the data, and wrote the manuscript; C.R. improved the language of the manuscript; W.W., C.R., L.D., and S.Y. gave advice and edited the manuscript. All authors read and approved the final manuscript.

\section{Conflict of interest}

The authors declare that they have no conflict of interest.

Supplementary Information accompanies this paper at (https://doi.org/ 10.1038/s41438-020-00377-y).

Received: 30 November 2019 Revised: 23 June 2020 Accepted: 7 July 2020 Published online: 01 October 2020

\section{References}

1. Luo, Y., Zeng, K. \& Ming, J. Control of blue and green mold decay of citrus fruit by pichia membranefaciens, and induction of defense responses. Sci. Hortic. 135, 120-127 (2012).

2. Yun, Z. et al. Sweating treatment enhances citrus fruit disease resistance by inducing the accumulation of amino acids and salicylic acid-induced resistance pathway. Physiol. Plant 155, 109-125 (2015).

3. Smilanick, J. L., Mansour, M. F., Gabler, F. M. \& Sorenson, D. Control of citrus postharvest green mold and sour rot by potassium sorbate combined with heat and fungicides. Postharvest Biol. Technol. 47, 226-238 (2008).

4. Schirra, M., D'Aquino, S., Cabras, P. \& Angioni, A. Control of postharvest diseases of fruit by heat and fungicides: efficacy, residue levels, and residue persistence. J. Agric. Food Chem. 59, 8531-8542 (2011).

5. Mckay, A. H., Förster, H. \& Adaskaveg, J. E. Toxicity and resistance potential of selected fungicides to Galactomyces, and Penicillium, spp. causing postharvest fruit decays of citrus and other crops. Plant Dis. 96, 87-96 (2012).

6. Zhu, F. et al. Salicylic acid treatment reduces the rot of postharvest citrus fruit by inducing the accumulation of $\mathrm{H}_{2} \mathrm{O}_{2}$, primary metabolites and lipophilic polymethoxylated flavones. Food Chem. 207, 68-74 (2016).

7. Vlot, A. C., Dempsey, D. A. \& Klessig, D. F. Salicylic acid, a multifaceted hormone to combat disease. Annu. Rev. Phytopathol. 47, 177-206 (2009).

8. Spoel, S. H. \& Dong, X. How do plants achieve immunity? Defence without specialized immune cells. Nat. Rev. Immunol. 12, 89-100 (2012).

9. Chen, Z. et al. Biosynthesis of salicylic acid in plants. Plant Signal. Behav. 4, 493-496 (2009).

10. Loake, G. \& Grant, M. Salicylic acid in plant defense-the players and protagonist. Curr. Opin. Plant Biol. 10, 466-472 (2007).

11. Zhang, Y., Chen, K., Zhang, S. \& Ferguson, I. The role of salicylic acid in postharvest ripening of kiwifruit. Postharvest Biol. Technol. 28, 67-74 (2003).

12. Yao, H. \& Tian, S. Effects of pre- and post-harvest application of salicylic acid or methyl jasmonate on inducing disease resistance of sweet cherry fruit in storage. Postharvest Biol. Technol. 35, 253-262 (2005).
13. Sayyari, M., Babalar, M., Kalantari, S., Serrano, M. \& Valero, D. Effect of salicylic acid treatment on reducing chilling injury in stored pomegranates. Postharvest Biol. Technol. 53, 152-154 (2009).

14. Mohammadreza, A. \& Aghdam, M. S. Impact of salicylic acid on post-harvest physiology of horticultural crops. Trends Food Sci. Technol. 21, 502-509 (2010).

15. Zhou, Y. H. et al. Effect of Pichia membranaefaciens in combination with salicylic acid on postharvest blue and green mold decay in citrus fruits. Biol. Control 74, 21-29 (2014)

16. Rushton, P. J., Somssich, I. E., Ringler, P. \& Shen, Q. J. WRKY transcription factors. Trends Plant Sci. 15, 247-258 (2010).

17. Wang, F. P., Zhao, P. P., Zhang, L. \& Du, Y. P. Functional characterization of WRKY46 in grape and its putative role in the interaction between grape and phylloxera (Daktulosphaira vitifoliae). Hortic. Res. 6, 102 (2019).

18. Chen, F. et al. The WRKY transcription factor family in model plants and crops. Crit. Rev. Plant Sci. 36, 1-25 (2018).

19. Andreasson, E. et al. The MAP kinase substrate MKS1 is a regulator of plant defense responses. EMBO J. 24, 2579-2589 (2014).

20. Kim, K. C., Lai, Z. B., Fan, B. F. \& Chen, Z. X. Arabidopsis WRKY38 and WRKY62 transcription factors interact with histone deacetylase 19 in basal defense. Plant Cell 20, 2357-2371 (2008)

21. Liu, X., Bai, X., Wang, X. \& Chu, C. OsWRKY71, a rice transcription factor, is involved in rice defense response. J. Plant Physiol. 164, 0-979 (2007).

22. Fan, B. et al. Roles of Arabidopsis WRKY3 and WRKY4 transcription factors in plant responses to pathogens. BMC Plant Biol. 8, 68 (2008).

23. Qiu, D. et al. OsWRKY13 mediates rice disease resistance by regulating defense-related genes in salicylate- and jasmonate-dependent signaling. Mol. Plant Microbe Interact. 20, 492 (2007).

24. Bhattarai, K. K. et al. WRKY72-type transcription factors contribute to basal immunity in tomato and Arabidopsis as well as gene-for-gene resistance mediated by the tomato R gene Mi-1. Plant J. Cell. Mol. Biol. 63, 229-240 (2010).

25. Kim, C. Y. et al. Functional analysis of a cold-responsive rice WRKY gene, OsWRKY71. Plant Biotechnol. Rep. 10, 13-23 (2016).

26. Xing, D. H. et al. Stress- and pathogen-induced Arabidopsis WRKY48 is a transcriptional activator that represses plant basal defense. Mol. Plant 1, 459-470 (2008)

27. Chujo, T. et al. OsWRKY28, a PAMP-responsive transrepressor, negatively regulates innate immune responses in rice against rice blast fungus. Plant Mol. Biol. 82, 23-37 (2013).

28. Zhang, Z. L. et al. A negative regulator encoded by a rice WRKY gene represses both abscisic acid and gibberellins signaling in aleurone cells. Plant Mol. Biol. 70, 139-151 (2009).

29. Cao, J. et al. Effects of postharvest salicylic acid dipping on Alternaria rot and disease resistance of jujube fruit during storage. J. Sci. Food Agric. 93, 3252-3258 (2013).

30. Ament, K. et al. Methyl salicylate production in tomato affects biotic interactions. Plant J. 62, 124-134 (2010).

31. Zhao, N. et al. Biosynthesis and emission of insect-induced methyl salicylate and methyl benzoate from rice. Plant Physiol. Biochem. 48, 279-287 (2010).

32. Park, S. W., Kaimoyo, E., Kumar, D., Mosher, S. \& Klessig, D. F. Methyl salicylate is a critical mobile signal for plant systemic acquired resistance. Science $\mathbf{3 1 8}$ 113-116 (2007).

33. Boatwright, J. L. \& Pajerowska, M. K. Salicylic acid: an old hormone up to new tricks. Mol. Plant Pathol. 14, 623-634 (2013).

34. Raikhel, N. Nuclear targeting in plants. Plant Physiol. 100, 1627-1632 (1992).

35. Laskey, R. A. \& Dingwall, C. Nuclear shuttling: the default pathway for nuclear proteins? Cell 74, 585-586 (1993).

36. Cheng, M. N. et al. The WRKY transcription factor HpWRKY44 regulates CytP450-like1 expression in red pitaya fruit (Hylocereus polyrhizus). Hortic. Res. 4, 17039 (2017).

37. Hara, K., Yagi, M., Kusano, T. \& Sano, H. Rapid systemic accumulation of transcripts encoding a tobacco WRKY transcription factor upon wounding. Mol. Gen. Genet. 263, 30-37 (2000).

38. Dong, J., Chen, C. \& Chen, Z. Expression profiles of the Arabidopsis WRKY gene superfamily during plant defense response. Plant Mol. Biol. 51, 21-37 (2003).

39. Kalde, M. Barth, M. Somssich, I. E. \& Lippok, B. Members of the Arabidopsis WRKY group III transcription factors are part of different plant defense signaling pathways. Mol. Plant Microbe Interact. 16, 295-305 (2003).

40. Zeng, K. F., Cao, J. K. \& Jiang, W. B. Enhancing disease resistance in harvested mango (Mangifera indica L. Cv. 'Matisu') fruit by salicylic acid. J. Sci. Food Agric 86, 694-698 (2010). 
41. Lee, K. T. et al. Responses of transformed root culture of atropa belladonna to salicylic acid stress. J. Biosci. Bioeng. 91, 586-589 (2002).

42. Fukami, H. et al. Salicylic acid carboxyl methyltransferase induced in hairy root cultures of atropa belladonna after treatment with exogenously added salicylic acid. Plant Cell Physiol. 43, 1054 (2002).

43. Hu, Y., Dong, Q. \& Yu, D. Arabidopsis WRKY46 coordinates with WRKY70 and WRKY53 in basal resistance against pathogen Pseudomonas syringae. Plant Sci. 185-186, 0-297 (2012).

44. Li, J., Brader, G. \& Palva, E. T. The wrky70 transcription factor: a node of convergence for jasmonate-mediated and salicylate-mediated signals in plant defense. Plant Cell 16, 319-331 (2004).

45. Bekir, ülker \& Somssich, M. I. E. The WRKY70 transcription factor of Arabidopsis influences both the plant senescence and defense signaling pathways. Planta 226, 125-137 (2007).

46. Jiang, Y. Z. et al. Overexpression of Poplar PtrWRKY89 in transgenic Arabidopsis leads to a reduction of disease resistance by regulating defense-related genes in salicylate- and jasmonate-dependent signaling. PLOS ONE 11, e0149137 (2016).

47. Jiang, C. H. et al. Transcription factors WRKY70 and WRKY11 served as regulators in rhizobacterium Bacillus cereus AR156-induced systemic resistance to Pseudomonas syringae pv. tomato DC3000 in Arabidopsis. Proc. Prehist. Soc. 41, 156-253 (2016).

48. Deng, B. et al. Comparative RNA-seq analysis of citrus fruit in response to infection with three major postharvest fungi. Postharvest Biol. Technol. 146 134-146 (2018)

49. Bugos, R. C. et al. RNA isolation from plant tissues recalcitrant to extraction in guanidine. Biotechniques 05, 734-737 (1995).

50. Livak, K. J. \& Schmittgen, T. D. Analysis of relative gene expression data using real-time quantitative PCR and the $2^{-\Delta \Delta} \tau_{T}$ Method. Methods 25, 402-408 (2001).

51. Pan, X., Welti, R. \& Wang, X. Quantitative analysis of major plant hormones in crude plant extracts by high-performance liquid chromatography-mass spectrometry. Nat. Protoc. 5, 986-992 (2010). 